ceaseless urging. Her pulse this day was 130 to 140 per minute, and very thready; the tongue was covered with a thick brown coat; the mental faculties perfectly clear. The bowels acted after an enema ; urine scanty, loaded with lithates, but not dark or carbonaceous looking. This same day (December 7 th) the carbolic dressings were changed for linseed-meal poultices, and this had not been done many hours be. fore a change for the better showed itself. In the course of forty hours actual pus was present in the poultices, and the constitutional irritation was entirely removed, the sickness being the last to leave.

Our patient continued to do well for a week, gradually improving up to December 15 th, when the carbolic lotion (same strength) was reemployed. Within thirty-six hours vomiting began again, accompanied by the same train of symptoms as on the previous occasion. The discharge, which was abundant and purulent on the evening of the $15^{\text {th }}$, became scanty and rusty by the morning of the 17 th ; the urging, if possible, was worse than before.

Poultices were again substituted on the 18 th, when we almost had given up hoping to save the patient's life. She was entirely supported at this time by enemata, her stomach not being able to retain even a little beef-tea or brandy and soda. Two or three days after the change in the application, healthy pus in fair quantity was coming from the elbow ; and, with the exception of great prostration, the evil symptoms had all abated. Our suspicions were now awakened as to the existence of some undoubted relation between the above symptoms and the nature of the dressing; yet our faith in carbolic acid was so strong, that we hardly dared suspect it as the cause.

The patient went on very well with the use of poultices up to Decem. ber 27 th, when, for the last time, and with a similar result, the carbolic lotion was resumed. The wound was now nearly healed; two sinuses which had formed we thought to benefit by a bi-daily injection of the foregoing lotion. Fortunately, the first appearance of suspicious signs (i.e., slight sickness after food) found us on the alert, and the injections were at once discontinued, the mischief being thereby nipped in the bud. For the next three weeks warm-water dressing constituted the sole treatment, and the patient left for her home on January 22nd, 1869, quite cured; with an arm which has since become fairly useful.

REMARKS. - Several instances of carbolic poisoning are on record, two if not three having had a fatal result. One appeared in the general and medical papers about two years ago. A man suffering from tooth. ache sought to relieve it by inhaling the acid, and died from the effects. Then there is the more recent case at the Worcester Infirmary-this, too, proving rapidly fatal - the cause being a carbolic enema. Even during the administration a fit occurred, accompanied by stertorous breathing, death resulting in twenty minutes. About the same time an account appeared of three unfortunate itch-patients who were admitted into a workhouse near Birmingham, and, through the mistake of a nurse, were treated with a strong solution of impure phenic acid applied freely to the surface of the body. One of the three, I believe, died from its effects, the other two suffering severely. Another case of partial poisoning was alluded to in a Guy's Hospital report as having occurred to Dr. Wilks, who saw the patient in consultation with Mr. Hill of Camberwell. Through the kindness of the latter gentleman, I have been favoured with the following brief history. "The case to which you refer was one of typhoid fever, in which extensive bed-sores existed. For two days I dressed them with lint soaked in carbolic acid (one part to five of linseed-oil). After the second dressing, severe and continuous vomiting occurred, and the urine became very dark (as with anasarca after scarlatina), leading me at first to think that there was hæmorrhage from the kidney. On discontinuing the carbolic dressing, the vomiting ceased and the urine became natural. Under the microscope there were no signs of blood-corpuscles, only dark amorphous particles, as of carbon. The sores were afterwards dressed with Condy's fluid (one to sixteen water), which removed the odour. They ultimately healed, though the worst that I have seen." In the University College Hospital Report, by Mr. Cluff, it is stated "that more cases of persistent vomiting of an uncontrollable nature occur after operations, which vomiting may or may not be aided by chloroform, and the only cure for which is the leaving off of the carbolic applications." In the three fatal instances, the acid seems to have acted as a narcotico-irritant, this action being probably due either to the strength or the manner in which it was given ; but in Mr. Hill's case, as in my own, an irritant effect only was noticed. The same appears to hold good in Mr. Cluff's experience. The terrible vomiting is deserving of special notice. In Miss J.'s case every thing was tried to check it, save ice, which we could not procure at the time when most wanted. Creasote was given in pill before we suspected the real cause of the emesis ; and it is a noteworthy fact that, notwithstanding the close relation between this drug and carbolic acid, it did not aggravate the urging tendency in the least, though quite ineffectual as regards stop. ping it. The only means of relief we found-and, fortunately, a sure one-was that advised by Mr. Cluff; viz., at once to leave off all carbolic appliçations.

In conclusion, I would briefly allude to the following points in connection with our patient. I. The very small quantity of acid which was used (to this, perhaps, the absence of carbon in the urine may have been due); 2. The close resemblance between the bad effects of the acid and the symptoms of pyæmic poisoning, the distinctive marks being the absence of wandering, icteric tint, and sweet breath ; lastly, and what is most conclusive, the intermittent character of the illness, so closely corresponding with the alternating use and disuse of carbolic acid; 3. Chloroform can hardly be blamed for aiding in the dangerous turn which our case took, seeing that our patient went on most satisfactorily for the first three days after the operation.

\section{CLINICAL LECTURE ON THE TREATMENT OF STRICTURE.}

\section{BY Mr. PAGET, F.R.S.}

Mr. PAGET lectured on two cases of retention of urine with slight stricture, in which the patients had been treated in the first instance by warm bath, laudanum, and rest, without catheterism, the last measure having been reserved until many days after the retention was relieved, and employed for the treatment of the purely organic stricture only. In one of the cases (to which Mr. Paget referred in a former clinical lecture, see p. 208), catheterism was attempted on admission, for a short time only, but unsuccessfully ; this man had had incontinence of urine for six weeks before he got retention, and yet, after three weeks' treatment, by rest, alkalies, and plain diet, Mr. Paget was able to pass No. 9 without any difficulty into his bladder.

In the other case, retention was complicated by a perinæal abscess, which was opened. The man was treated without any attempt at catheterism until the third week, when a single unsuccessful attempt was made ; but a few days later Mr. Paget was able to pass No. 6 at once into the bladder. Mr. Paget dilated on the advantages of rest, the warm bath, and opium, in relieving retention, and on the continuance of rest with alkaline medicines and unstimulating diet for the subsequent complete removal of spasm, and of that swelling of the urethral mucous membrane which may not only produce retention, but, as in the first patient mentioned, may cause symptoms closely simulating those of the worst forms of organic stricture. The chronic congestion of the urethral mucous membrane, which probably existed in that patient and caused incontinence, was compared by the lecturer with chronic conjunctivitis and chronic swelling of the nasal mucous membrane.

Mr. Paget considered that the perinæal abscess, which formed in the second patient, was probably the result of inflammation in strictures contiguous to the urethra, but without any extravasation of urine. Many of the perinæal abusesses formed in connection with retention were produced in this way, and not, as is commonly taught, by ulceration of the urethra and localised extravasation.

Mr. Paget went on to remark on the morbid sympathies of the urethra, sympathies which were found in no other mucous track, and were quite peculiar to that over which the urine passed from the bladder. The first of these peculiar manifestations of sympathy with dis. tant parts was the syncope which occasionally follows the passage of a catheter, the next was epileptiform convulsion from the same cause. Mr. Paget remarked that these two results never happened unless the patient were standing up, just as the same phenomena after excessive blood-letting were noticed only when the patient was in the erect position. Thirdly, rigor following the passage of instruments into the bladder was mentioned; this result was commonest in patients who had never been catheterised before, and unlike the two other phenomena, did not come on until water was passed for the first time after the use of the catheter. Riyor after catheterism was said to be commonest in persons who had ague, or who had lived in'tropical countries, and the phenomena of the shivering fit were described as corresponding exactly with the course of an attack of ague. The rigor might often be prevented by the administration of brandy and water or other alcoholic stimulant immediately after the operation; and Mr. Paget spoke in high terms of Sir B. Brodie's practice in this respect.

In referring to the occasionally very dangerous character of these shivering fits, Mr. Paget mentioned that he had known death to follow simply sounding for stone in no less than six cases. As a rule, howcver, a rigor occurring soon after lithotrity, lithotomy, or the passage of a catheter, was not a dangerous sign. This brought the lecturer to 
his fourth peculiar sequence of catheterism and lithotrity; viz., the occasional occurrence of pyæmia, after a rigor, apparently from simple irritation of the urethra, and without any injury to its structure or effu. sion of urine. Mr. Paget then referred to another of these curious relations between the urethra and distant parts, urethral rheumatism (including the so-called gonorrhoal rheumatism), an affection which, if it could not be described as pyæmic, yet certainly presented many points of close resemblance to pyæmia.

Mr. Paget was of opinion that any inflammation of the urethra, whether contagious or not, might be followed by the swelling and effusion into the joints which characterises urethral rheumatism. Lastly, Mr. Paget mentioned the connection which he believed to exist between certain cases of sclerotitis inflammation of the urethra and effusion into joints, inclining to the belief that patients in whom these conditions coexisted were often to be described as gouty rather than rheumatic.

\section{ABSTRACT OF A CLINICAL LECTURE.}

\section{BY MR. ERICHSEN.}

THE following is an abstract of Mr. Erichsen's lecture delivered at University College Hospital on March 21st.

Case of Aneurism of the Profunda Femoris, cured by Compression in twenty-four hours. - There is a case of considerable interest now in the Hospital, of which I shall say more on a future occasion, but wish now to draw your attention to some of its more characteristic features. It is that of a large aneurismal tumour at the upper part of the right thigh. The history is obscure. Last Christmas the patient had an attack of pneumonia, on which a period of bad health supervened, and soon this swelling in the thigh made its appearance. On admission, we found a large tumour in the abovementioned situation; there was no thrill, no pulsation, in it. The superficial femoral artery crossed it on its inner aspect; and the pulsation in this, and in its branches below the tumour, viz., popliteal and tibial, was quite normal. In consequence of the stretching and compression of the femoral vein, there was some cedema of the lower part of the leg. I had then some doubts as to its nature. It might have been, first, an aneurism undergoing consolidation; secondly, a deep abscess beneath the periosteum ; thirdly, a tumour springing from bone. At the next visit I found the tumour increased in size considerably; it was pulsating strongly, the pulsation being heaving rather than expansile. There were a distinct thrill at the inner part and a bruit, both synchronous with the entrance of blood into the common femoral, and arrested by compression of the same. The muscles of the anterior part of the thigh were stretched and separated. As to its nature, the first idea was that the tumour was an aneurism, but scarcely of the superficial femoral artery, for this was pulsating over the tumour for its whole length, and even below it, and the pulsation in the popliteal artery was quite free. Now, aneurism of the superficial femoral springs from the fore part of the artery. In my experience I never knew a case where it sprang from behind. This idea was therefore set aside. Secondly, the idea that it was a pulsating tumour of bone springing from the anterior and inner aspect of the femur, was favoured by $(a)$ the strong pulsation without great expansion; (b) the distinct thrill; (c) the harsh bruit, so commonly heard in aneurism by anastomosis consisting in a congeries of smaller vessels. Thirdly, aneurism of the profunda femoris is probably the correct solution of the case. This is a very rare disease, there being few cases recorded. In my own experience I have met with but one instance. A man was under the care of one of the physicians in this Hospital for pneumonia: he complained of some swelling and uneasiness in the thigh, but such was the gravity of his internal complaint that no heed was paid to this. He died. At the post mortem examination, a large circumscribed false aneurism of the profunda artery of the thigh (of the size of a cocoa-nut) was discovered. I was disposed, then, to consider this an aneurism of the profunda artery, because the aneurism was unquestionably in the situation of this artery, being between the superficial femoral artery and the bone. That the latter vessel was quite free, is proved by the fact that the pulsation, through it and its terminal branches, was quite normal in character. Again, pressure on the common femoral arrested the pulsation, the bruit, the thrill, and even the size of the tumour, so there could only be one other vessel than the superficial femoral to supply it; viz., the profunda.

The Influence of Treatment. - Pressure on the common femoral artery arresting the signs, Carte's compressor was applied. The patient bore this badly ; he was irritable, and writhed from under the com. pressor. In the evening, forty grains of hydrate of chloral were given. With this he slept heavily and deeply for twelve hours, during which time the compressor was kept in firm application. In the morning, the pulsation and thrill were found much diminished, the brait less marked, and there was decided consolidation in the tumour. He could no longer bear this instrument; but, for the next twelve hours, digital compression was used and arrested all the signs. For safety, pressure on the artery was maintained for some hours afterwards; but the twenty-four hours' compression did the work entirely. This would lead us to infer that the tumour was a real aneurism not connected with bone. The thigh has decreased in circumference an inch and a half. All the aneurismal signs are removed, which could only happen in the case of a real aneurism. I am inclined to the opinion that the tumour was consolidating on admission, but that the manipulation used in the examination and diagnosing of it set the aneurism going again-and that then compression succeeded in curing it.

Reference to the anatomy of the profunda makes it easy to see that compression should cure aneurismal disease of it. Being destined chiefly for the nutrition of the thigh, it has a short trunk, and speedily breaks up into minor branches: hence there is not a long trunk going through the aneurism, and the blood has not to traverse the sac from the vessel by which it enters to one large vessel of exit, but to filter through a number of smaller vessels; consequently the coagulation of the blood is very much favoured. We cannot prophesy as to the future and the complications of the case : the tumour may undergo disorganisation and suppuration; if not, it will probably advance by steady progress to recovery.

Mr. Erichsen then proceeded to consider a case of Perforating Ulcer of the Foot.-These cases are unusual, and of great practical interest. In this we observe the remains of a hard corn on the sole of the foot, while on the corresponding part of the dorsum is a small ulcerated aperture, from which pus is discharged. This ulcer is situate between the third and fourth metatarsal bones, very far back. On introducing a probe into the dorsal wound, it makes its exit in the sole through an aperture in the centre of the corn, there being a sinus which perforates the foot. The probe passes close to the bone (the neck of the third metatarsal), but no necrosis nor caries is felt. Here, then, we have a sinus perforating the foot in a man otherwise healthy, there being no signs of struma or any constitutional disease. How is it to be explained? It appears that he first noticed the corn about eighteen months ago. It is situate between the third and fourth metatarsal bones. Suppuration took place in the subcutaneous cellulo-adipose tissue here, and the pus escaped through an aperture in the centre of the corn. This aperture subsequently closed, and the pus, travelling upwards, finally made its exit through the integumental structures of the dorsum of the foot.

Treatment. - If there be diseased bone, remove it. In this case there is none, so we adopt the same line of practice as in any sinus, viz., stimulation. A seton of two silk threads is introduced, which keeps up a free opening in the sole, and at the same time stimulates the sinus by the irritation set up by its presence.

\section{THERAPEUTICAL MEMORANDA.}

\section{THE TREATMENT OF DIPHTHERIA.}

By George Hill, M.D.Edin., Hooton, Chester.

AN epidemic of diphtheria having prevailed in this neighbourhood recently, I have had an opportunity of testing the therapeutic value of various topical remedies. I will not occupy space with the details of illustrative cases, but will state my conclusions briefly.

Lactic acid, as recommended by MM. Bricheteau and Adrian, is an invaluable application, dissolving the diphtheritic membrane in a wonderful manner. It must be freely and repeatedly applied, and, being a harmless agent, it may be used with safety by those in charge, in the absence of the practitioner. But it must not be solely depended on, as it seems to possess no power of preventing the re-formation of the membrane. Of the other topical remedies, I found none so efficacious as the liquor ferri perchloridi fortior of the Pharmacopocia. A gargle of dilute hydrochloric acid, two or three drachms to eight ounces, is of great service in clearing the mouth and throat of the viscid mucus which accumulates with such rapidity. I also found an occasional gargle of carbolic acid, two or three grains to the ounce, of use in destroying fotor and cleansing the mouth. Carbolic acid inhalations seem good in theory, and it is possible that they may be of service in freeing the system from the diphtheritic poison. But the local symptoms were not improved by their use, and if, continued long, they seemed to aggravate the odema, which adds so much to the distress of the patient. Of internal remedies I have not much to say. I prescribe a drachm of chlorate of potash, in a pint of water, with lemon-juice, to be used as a common drink daily. 